\title{
Intracapsular cataract extraction for the management of dense cataracts and zonular weakness.
}

\author{
Steven B Koenig* \\ Department of Ophthalmology, Medical College of Wisconsin, Milwaukee, USA
}

\begin{abstract}
Purpose: To describe the use of Intracapsular Cataract Extraction (ICCE) with and without concomitant Pars Plana Vitrectomy (PPV) for the treatment of selected cases of dense cataract and zonular weakness.

Case Series: This is a retrospective analysis of five eyes of four patients undergoing ICCE during the last eight years.

Results and discussion: Five eyes of four patients underwent ICCE. Two patients had traumatic cataracts and amblyopia, one patient ( 2 eyes) had ectopia lentis associated with homocystinuria. All eyes had dense nuclear sclerotic, mature or hypermature cataracts in addition to generalized zonular weakness. Three eyes underwent combined PPV. One eye underwent concomitant repair of a rhegmatogenous retinal detachment. Three eyes received anterior chamber intraocular lenses. All surgeries were successfully performed without complication, although one eye developed reversible cystoid macular edema following surgery. Three eyes without pre-existing amblyopia experienced excellent post-operative visual acuity (range 20/20 to 20/30) during an average follow-up period of 17 months.

Conclusion: ICCE alone or in combination with pars plana vitrectomy is a safe and effective method for removing dense cataracts in the setting of zonular weakness or absence.
\end{abstract}

Keywords: ICCE, Zonular weakness.

Accepted on April 12, 2019

\section{Introduction}

ICCE is a surgical technique that removes the cataractous crystalline lens and its surrounding lens capsule. The procedure requires anesthesia and akinesia of the globe, a large corneoscleral incision, zonulysis, and a cryoprobe to extract the lens [1]. Due to delayed wound healing, slow visual recovery, lack of capsular support for a posterior chamber intraocular lens and greater risk of complications, the procedure has largely been abandoned. However, ICCE may be helpful in selected eyes with densely brunescent, mature or hypermature cataracts with dense nuclei in the setting of zonular absence or weakness [2]. In such cases, ICCE with or without an associated vitrectomy may reduce the risk of lens dislocation possibly associated with manual extracapsular surgery (ECCE) and avoid the excessive ultrasound energy associated pars plana phacofragmentation of a hard lens in the posterior segment [3]. The purpose of this paper is to present clinical scenarios in which ICCE may be the preferred technique for lens removal.

\section{Case Series}

This study was approved by the Investigational Review Board (IRB) of the Medical College of Wisconsin. A retrospective review of all patients undergoing either ICCE alone or ICCE together with PPV was performed over an eight year period (2010-2018). Clinical information included the age and sex of the patient, the cause of zonular weakness, baseline visual acuity, slit lamp findings and the indication for surgery. Intraoperative and post-operative complications as well as final visual acuity, post-operative interval and additional treatment were recorded.

\section{Surgical technique}

In each case, ICCE was performed under local anesthesia using an O'Brien and retrobulbar block of $2 \%$ lidocaine [1]. A superior rectus bridal suture was used to rotate the globe inferiorly. A fornix based conjunctival flap was prepared with Westcott scissors and .12 Calibri forceps. A $12 \mathrm{~mm}$ corneoscleral groove was made with a razorblade knife preset to 500 microns in depth. The wound was extended with left and right-handed scissors after creating a small paracentesis with a 15 degree blade at 12 o'clock. An 8-0 polyglactin suture (Ethicon) was passed through the 12 o'clock lip of the cornea, tied upon itself and retracted by an assistant surgeon in order to expose the iris and anterior surface of the lens. A peripheral iridotomy was performed with Vannas scissors. After drying the surface of the cataract, a cellulose sponge was used to retract the superior iris. A Frigitonics cryoprobe (Trumbull, CT) was placed on the superior half of the lens and an ice ball created by activating the freezing element. Once an adequate ice ball formed, the lens was gradually delivered by gentle rotational and rocking movements being careful not to freeze the iris or corneal endothelium. The wound was then temporarily closed with three interrupted 8-0 polyglactin 
Citation: Koenig SB. Intracapsular cataract extraction for the management of dense cataracts and zonular weakness. J Clin Ophthalmol 2019;3(1):137-40.

sutures and an air bubble placed in the anterior chamber following injection of a small amount of acetylcholine chloride to constrict the pupil. The wound was then closed with multiple interrupted $10-0$ nylon sutures replacing the temporary polyglactin sutures (Ethicon) in the process. Following removal of the superior rectus suture, the conjunctival flap was sealed with wet field cautery. The eye was treated with topical corticosteroid drops four times daily for one month followed by gradual taper over the following month. Topical antibiotics were applied for one week. Topical glaucoma meds and/or oral acetazolamide were used as necessary to control intraocular pressure postoperatively. A combined 23-gauge cannulated three port PPV was performed by a vitreoretinal surgeon prior to cataract extraction in order to reduce the risk of vitreous loss or vitreous traction (Cases 2, 4). If indicated, an anterior chamber intraocular lens was placed under viscoelastic using a Sheets glide. The viscoelastic was carefully removed using a Simcoe cannula at the end of the procedure.

\section{Case 1}

The patient is a 78 year old male with a history of perforating corneal injury to the right eye as a child. In addition to a dense corneal stromal scar, the patient had a history of traumatic cataract, amblyopia, and sensory exotropia with an associated loss of vision of 20/400. He presented to our institution complaining of severe right sided pain, nausea and foreign body sensation. His examination was remarkable for best spectacle corrected visual acuity of light perception, OD and 20/25, OS. The intraocular pressure measured $33 \mathrm{~mm} \mathrm{Hg}$, OD and $11 \mathrm{~mm} \mathrm{Hg}$, OS. Slit lamp examination of the right eye revealed mild conjunctival injection, a dense corneal stromal scar at 9 o'clock with microcystic epithelial edema and folds in Descemet's membrane. The anterior chamber was open with +2 cell and flare. The hypermature cataract exhibited a wrinkled anterior lens capsule, dense nuclear sclerosis and moderate phacodonesis. There was no view of the posterior pole of the right eye. B-scan ultrasonography demonstrated no retinal detachment or choroidal mass. The patient was felt to have phacolytic glaucoma and was initially treated with topical brimonidine tartrate $0.2 \%$, timolol maleate $0.5 \%$ and acetazolomide $250 \mathrm{mg}$ po q.i.d. prior to undergoing ICCE without placement of an intraocular lens. His post-operative course was initially characterized by moderately elevated intraocular pressure which was eventually controlled with brimonidine, timolol, and dorzolamide. The vision improved to hand motions.

\section{Case 2}

The patient is an 83 year old woman with a complicated past ocular history including trauma to the right eye as a child resulting in a cataract and amblyopia. She was taking latanoprost for elevated intraocular pressure in the right eye. She had a history recurrent retinal detachment in the left eye and dry age-related macular degeneration in both eyes. Upon presentation, the best spectacle corrected visual acuity was hand motions, OD and 20/150, OS. The right pupil revealed a traumatic sector iridectomy. The intraocular pressures were 15 $\mathrm{mm} \mathrm{Hg}$, OD and $18 \mathrm{~mm} \mathrm{Hg}$, OS. Slit lamp examination revealed a dense corneal stromal scar temporally in the right eye. The anterior chamber was deep. A large temporal sector iridectomy exposed the equator of the densely brunescent lens with absent zonules. Vitreous was present around the equator of the lens which demonstrated moderate phacodonesis. The patient underwent combined PPV with ICCE and placement of an anterior chamber lens implant (MTA3UO, Alcon Laboratory, Fort Worth, TX). The patient's post-operative course was unremarkable. Five months after surgery her best spectacle corrected visual acuity improved to 20/400 in the right eye, but was limited by underlying amblyopia, corneal scarring, and dry age-related macular degeneration. Normal intraocular pressures were maintained with latanoprost.

\section{Case 3}

This patient is a 68 year old woman who complained of gradual progressive loss of visual acuity in both eyes. Her past ocular history was negative. Her past medical history was remarkable for atherosclerotic cardiovascular disease, hypertension and non-insulin dependent diabetes mellitus. Her examination demonstrated best spectacle corrected visual acuity of hand motions in each eye. There was no afferent pupillary defect present. The intraocular pressure was $14 \mathrm{~mm}$ $\mathrm{Hg}$, OD and $12 \mathrm{~mm} \mathrm{Hg}$, OS. Slit lamp examination demonstrated clear corneae and deep and clear anterior chambers in both eyes. Each eye demonstrated a hypermature cataract with dense brunescence and marked phacodonesis. No vitreous prolapse was noted. B-scan ultrasonography demonstrated no retinal detachment or choroidal mass in either eye. Due to the risk of lens dislocation, the patient underwent ICCE with anterior chamber lens implantation in the left eye (MTA4UO, Alcon Laboratory, Fort Worth, TX). Her postoperative course was complicated by cystoid macular edema which was treated with topical prednisolone acetate and ketorolac four times daily with eventual resolution. Eight months after surgery, the best corrected spectacle visual acuity was $20 / 30+1$ in the left eye.

\section{Case 4}

This patient is a 52 year old male with a history of homocystinuria and bilaterally dislocated crystalline lenses. He complained of significant glare in both eyes and a new onset floater in his left eye. Ocular examination demonstrated a best corrected visual acuity of 20/30, OD and 20/30 -2, OS. With brightness acuity testing his vision dropped to 20/400 OU. Slit lamp examination of each eye revealed a clear cornea, deep anterior chamber and nearly black crystalline lenses (cataracta nigrans) which were markedly dislocated inferiorly. Vitreous was herniated around the superior pole of each cataractous lens. Fundus exam of the left eye demonstrated a temporal horseshoe tear with surrounding retinal detachment. The patient underwent combined PPV, laser endophotocoagulation, air fluid exchange, injection of sulfur hexafluoride gas and ICCE without placement of an anterior chamber lens implant in the left eye first. His post-operative course was uncomplicated and he elected to undergo combined PPV, ICCE and placement of an anterior chamber lens implant (MTA3UO, 
Alcon Laboratory, Fort Worth, TX) in the right eye $2^{1 / 2}$ years later understanding the need to wear an aphakic contact lens in the left eye to maintain binocular vision. Five months after surgery, the visual acuity measured 20/20 -2 in the right eye; after nearly three years, the vision was 20/20 -1 in the left eye. Intraocular pressures were normal and slit lamp exam demonstrated clear corneae with a well-positioned anterior chamber lens implant in the right eye and an aphakic anterior segment in the left eye.

\section{Results}

This study included five eyes of two men and two women. The average age was 70 years old. Two patients had an underlying history of trauma during childhood and had co-existing amblyopia (Cases 1, 2). Two patients had hypermature cataracts with dense nuclei, liquefied cortex and wrinkled anterior lens capsules, while three eyes had dense nuclear sclerotic lenses including two eyes with cataracta nigrans. All lenses were felt to be too dense to undergo phacoemulsification or phacofragmentation. All eyes had generalized zonular weakness with marked phacodonesis; three eyes had vitreous prolapse around the equator of the lens (Cases 2, 4). All eyes underwent successful removal of the cataract with no vitreous loss during lens delivery. Three eyes (Cases 2, 4) required combined PPV to remove vitreous around the lens prior to ICCE. Three eyes regained visual acuity between 20/20 -1 and 20/30. Two cases $(1,2)$ had preexisting co-morbidities (agerelated macular degeneration and/or amblyopia) that limited post-operative visual acuity. Two eyes required no treatment for glaucoma. One eye (Case 1) required maximal topical therapy for control of the intraocular pressure following phacolytic glaucoma.

\section{Discussion and Conclusion}

Subluxated or loose crystalline lenses may be seen in a variety of disorders including Marfan's syndrome, pseudoexfoliation syndrome, homocystinuria, Weil Marchesani syndrome, hyperlysinemia, sulfite oxidase deficiency and trauma [4]. Removal of the crystalline lens is usually performed when the patient develops unacceptable loss of vision, anisometropia, lens induced glaucoma, monocular diplopia or glare. The surgical management of a cataract with zonular weakness depends upon the surgeon's training, the extent of zonular loss, the density of the cataract, the position of the lens and the clarity of the media.

Phacoemulsification with posterior chamber lens implantation may be performed even in the setting of weak or missing zonules [5]. In eyes with localized or mild generalized zonular weakness, phacoemulsification may be completed using capsule support hooks and/or a capsular tension ring [6]. In eyes with more advanced zonular weakness, a modified capsular tension ring may be sutured to the sclera [7]. In cases of vitreous prolapse around the equator of the lens, a limited anterior vitrectomy or pars plana vitrectomy may reduce the risk of inadvertent vitreoretinal traction during phacoemulsification. 8 In eyes with more profound zonular loss or in subluxated lenses, combined PPV and lensectomy using pars plana phacofragmentation may be performed together with placement of either an anterior chamber lens implant, iris sutured posterior chamber lens implant or a scleral fixated posterior chamber lens using a variety of fixation techniques $[8,9]$. This approach provides the safety of a closed surgical system and allows for retrieval of the lens or lens fragments from the posterior segment if necessary [8]. However, pars plana phacofragmentation of dense nuclei may be technically difficult to perform, require excessive ultrasound energy, could possibly result in thermal injury to ocular tissue, and may not be safe in a patient with an impaired view due to corneal opacification [3].

In eyes with extremely dense cataracts and severe generalized zonular weakness, ICCE may actually be a preferred technique for lens removal $[3,10]$. Historically, ICCE for subluxated lenses was associated with an unacceptably high rate of vitreous loss, retinal detachment, and $[3,4]$ glaucoma presumably due in part to inadvertent vitreous traction during lens delivery. However, older surgical series were performed before the advent of modern PPV techniques which enable the surgeon to identify and remove vitreous from around the crystalline lens prior to removal. In our patients with subluxated lenses and vitreous prolapse (Case 2, 4), PPV was performed prior to ICCE in order to reduce the risk of vitreoretinal traction. Removal of the vitreous may also facilitate placement of an anterior chamber lens implant, evacuation of residual viscoelastic from the anterior chamber, and reduce the risk of pupillary block glaucoma post operatively.

ICCE may also facilitate lens removal in eyes with corneal scarring or edema where visualization of intraocular structures may be compromised. Case 1 presented with phacolytic glaucoma years after a perforating ocular injury resulted in a traumatic cataract. In this case, the scar together with corneal stromal and microcystic epithelial edema interfered with visualization of the anterior segment and would have complicated an extracapsular cataract extraction or pars plana lensectomy approach. In such eyes with co-existing elevated intraocular pressure, pre-operative pressure reduction and/or gradual decompression of the anterior chamber may reduce the risk of a choroidal hemorrhage which may be greater in the setting of an open globe with a large corneal scleral incision $[1,11]$. Complete removal of the entire lens capsular complex with ICCE may also help in the control of intraocular pressure in cases of phacolytic glaucoma [12].

During ICCE, the entire crystalline lens is delivered from the eye using a cryoprobe which produces an ice ball on and within the cataractous lens. Historically, chemical zonulysis using alpha-chymotrypsin was performed prior to lens removal. This agent is no longer available commercially. However, all eyes in this series had extreme generalized zonular weakness or absence obviating the need for additional zonulysis.

For surgeons not trained to perform ICCE, the procedure can be challenging. It requires a retrobulbar block to achieve akinesia and anesthesia of the globe and orbicularis. It is helpful to have an assistant who can retract the cornea during 
Citation: Koenig SB. Intracapsular cataract extraction for the management of dense cataracts and zonular weakness. J Clin Ophthalmol 2019;3(1):137-40.

cryoextraction and irrigate balanced salt solution to defrost corneal or iris tissue inadvertently incorporated into the ice ball. Due to the large corneoscleral incision, the globe is open for an extended period of time, placing the eye at increased risk of expulsive hemorrhage. Wound healing is typically prolonged and frequently complicated by surgeon induced corneal astigmatism. Finally, ICCE removes all capsular support preventing the endocapcular fixation of the posterior chamber lens implant. Nevertheless, in eyes with extreme zonular weakness, ICCE may be the best approach to remove selected lenses too dense for phacoemulsification or pars plana phacofragmentation. In eyes with vitreous prolapse around the crystalline lens, ICCE may be combined with PPV to reduce vitreoretinal traction and facilitate placement of an anterior chamber lens implant with good visual results.

\section{Source of Funding}

This study was supported in part by a grant from Dr. Myrna Larson and Dr. Nelson A Moffat.

\section{Conflicts of Interest}

The author has no conflicts of interest to disclose.

\section{References}

1. Jaffe NS, Jaffe MS, Jaffe GF. Surgical Technique. In: Cataract surgery and its complications. C.V. Mosby Co., St. Louis. 1976;pp:31-82.

2. Cionni R, Osher R. Complications of phacoemulsification surgery. In: Management and care of the cataract patient. Blackwell Science Inc., Cambridge. 1992;pp:210-49.

3. Michels RG, Shackeh CDE. Vitrectomy technique for removal of retained lens material. Arch ophthal. 1977;95:1767-73.

4. Jarrett II WH. Dislocation of the lens. A study of 166 hospitalized cases. Arch Ophthal. 1967;78:289-96.

5. Cionni RJ, Osher RH. Endocapsular ring approach to the subluxated cataractous lens. J Cataract Refract Surg. 1998;24:245-9.
6. Blecher MH, Kirk MR. Surgical strategies for the management of zonular compromise. Curr Opin Ophthalmol. 2008;19:31-35.

7. Cionni RJ, Osher RH. Management of profound zonular dialysis or weakness with a new endocapsular ring designed for scleral fixation. J Cataract Refract Surg. 1998;24:1299-1306.

8. Kazem S, Wirostko WJ, Sanka S, et al. Combined pars plana lensectomy-vitrectomy with open loop flexible anterior chamber intraocular lens (AC IOL) implantation for subluxated lenses. Tr Am Oph Soc. 2000;98:247-51.

9. Wagener MD, Cox TA, Ariyasu RG, et al. Intraocular lens implantation in the absence of capsular support: A report by the American Academy of Ophthalmology. Ophthalmology. 2003;110:840-59.

10. Lee SB, Eong KG, Yong VS. Management of subluxated crystalline lenses with planned intracapsular cataract extraction and anterior chamber intraocular lens implantation. Singapore Med J. 1999;40:352-5.

11. Speaker MG, Guerriero PN, Met JA, et al. A case-control study of risk factors for intraoperative suprachoroidal expulsive hemorrhage. Ophthalmology. 1991;98:202-9.

12. Richter C, Epstein DL. Lens-induced open angle glaucoma. In: The Glaucomas. C.V. Mosby Co., St. Louis. 1989;pp:1017-26.

\section{*Correspondence to:}

Steven B Koenig, MD

Department of Ophthalmology

Medical College of Wisconsin

925 N 87th Street, Milwaukee, WI 53226, USA

Tel: 414-955-7842

Fax: 414-955-6300

E-mail: hhenrics@mcw.edu 